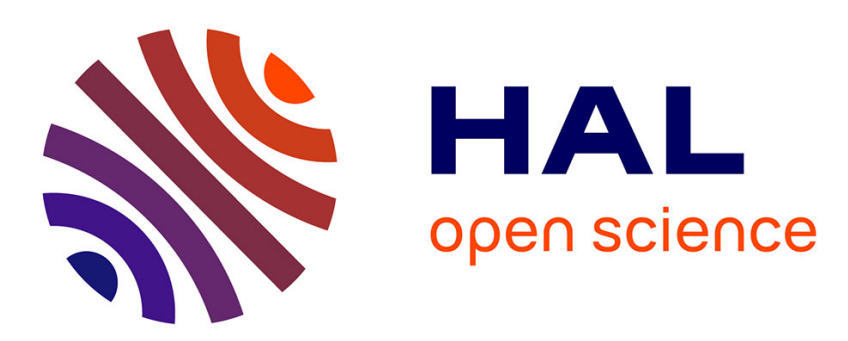

\title{
Stability analysis of the POD reduced order method for solving the bidomain model in cardiac electrophysiology
}

Cesare Corrado, Jamila Lassoued, Moncef Mahjoub, Nejib Zemzemi

\section{To cite this version:}

Cesare Corrado, Jamila Lassoued, Moncef Mahjoub, Nejib Zemzemi. Stability analysis of the POD reduced order method for solving the bidomain model in cardiac electrophysiology. Mathematical Biosciences, 2015, 10.1016/j.mbs.2015.12.005 . hal-01245685

\section{HAL Id: hal-01245685 \\ https://inria.hal.science/hal-01245685}

Submitted on 17 Dec 2015

HAL is a multi-disciplinary open access archive for the deposit and dissemination of scientific research documents, whether they are published or not. The documents may come from teaching and research institutions in France or abroad, or from public or private research centers.
L'archive ouverte pluridisciplinaire HAL, est destinée au dépôt et à la diffusion de documents scientifiques de niveau recherche, publiés ou non, émanant des établissements d'enseignement et de recherche français ou étrangers, des laboratoires publics ou privés. 


\title{
Stability analysis of the POD reduced order method for solving the bidomain model in cardiac electrophysiology
}

\author{
Cesare Corrado $^{\mathrm{a}}$, Jamila Lassoued ${ }^{\mathrm{b}, \mathrm{c}}$, Moncef Mahjoub $^{\mathrm{b}, \mathrm{c}}$, Néjib Zemzemi $^{\mathrm{a}, \mathrm{c}}$ \\ a INRIA, Bordeaux - Sud-Ouest, 200 Avenue de la vielle Tour 33405 Talence Cedex France. \\ ${ }^{b}$ University of Tunis El Manar, LAMSIN - ENIT, BP 37, 1002 Tunis Belvedere, Tunisia. \\ ${ }^{c}$ LIRIMA - Laboratoire International de Recherche en Informatique et Mathématiques \\ Appliquées.
}

\begin{abstract}
In this work we show the numerical stability of the Proper Orthogonal Decomposition (POD) reduced order method used in cardiac electrophysiology applications. The difficulty of proving the stability comes from the fact that we are interested in the bidomain model, which is a system of degenerate parabolic equations coupled to a system of ODEs representing the cell membrane electrical activity. The proof of the stability of this method is based an a priori estimate controlling the gap between the reduced order solution and the Galerkin finite element one. We present some numerical simulations confirming the theoretical results. We also combine the POD method with a time splitting scheme allowing a faster solution of the bidomain problem and show numerical results. Finally, we conduct numerical simulation in 2D illustrating the stability of the POD method in its sensitivity to the ionic model parameters. We also perform 3D simulation using a massively parallel code. We show the computational gain using the POD reduced order model. We also show that this method has a better scalability than the full finite element method.
\end{abstract}

Keywords:

Bidomain equation, reduced order method, proper orthogonal decomposition, stability analysis, a priori estimates, ionic parameters, Mitchell-Schaeffer model. 2000 Math Subject Classification: 34A12, 34A34, 35K20, 35B45, 35K57, 35K60, 35B65, 49M27 


\section{Introduction}

The electric wave in the heart is governed by a system of reaction-diffusion partial differential equations called bidomain model. This system is coupled nonlinearly to an ordinary differential equations (ODEs) modeling the cellular membrane dynamics (see $[5,27]$ ). The bidomain model is widely used in cardiac electrophysiology simulation. This mathematical model takes into account the electrical properties of the cardiac muscle.

Different numerical methods have been used for solving the bidomain model. Finite element method has been used in most of the works [35, 8, 10, 21, 4, 34, 14]. Some groups use finite difference method $[27,32]$ and others use finite volume method [9]. All these methods lead to a large linear system to solve, especially when using implicit schemes. Here we don't cite all the works that have been done in the literature, some other works could be found in the recent review of the bidomain model [15]. The computational cost of solving the bidomain problem becomes very important when we are interested in solving inverse problems [2]. Thus reducing the computational cost of the forward problem is a challenging issue. One of the most popular approaches used in model reduction is the Proper Orthogonal Decomposition (POD) method. This method was initially introduced for analyzing multidimensional data. In [26], Holmes et al. used this approach for understanding the turbulence flow phenomena. Until there, the POD was presented only as an efficient post-processing technique in order to extract coherent structures of data from numerical simulations or experiments. The proper orthogonal decomposition modes allow to provide basis functions that could be used to define a low-dimensional subspace on which one would project a linear system. This idea was first applied by Aubry et al. [23] to model the wall region of a turbulent boundary layer. More recently Delville et al. [11] used it to study the dynamics of coherent structures in turbulent mixing layer. Moreover, existing er-

ror estimates are expressed with respect to quantities which are not controlled in the construction of the POD basis [36]. In the literature, there are some a priori estimates results: Henri and Yvon [13] show an error estimate for continuous and linear parabolic problems. Kunisch and Volkwein [17] show a priori error estimates for a generic non linear parabolic evolution PDEs at the discrete level.

As concerns its use in the cardiac electrophysiology field, the reduced order modelling based on POD has been used by Chapelle et al. [7]. The authors demonstrate an a priori error estimates result that guaranties the stability of the POD method in case of a nonlinear parabolic wave-like equation where the nonlinear term is a cubic Lipschitz function. This model is known as the monodomain 
equation and allows to describe the propagation of the electrical wave but have some limitation in application like electrocardiograms modelling [3]. The use of the bidomain model is necessary in this case. Recent works have shown the usability and the efficiency of the reduced order modelling based on POD for the numerical simulation of electrocardiograms based on the bidomain model [4].

It has also been used for the ionic model parameter estimation but more interestingly for infarction localisation based on simulated data [2]. The application of reduced order is appealing because it allows to dramatically decrease the computational cost of the forward problem and consequently in the inverse problem. The problem is that for the bidomain model there is no study showing the stability of the reduced order method. The work by Chapelle et al. [7] does not cover the bidomain problem, the main difficulty comes from the fact that the bidomain model is degenerated. In this work we prove the stability of the POD method for the bidomain model based on an a priori error estimates and we illustrate the theoretical results by some numerical simulations.

In section 2 we present the mathematical model of the electrical activity in the heart based on the bidomain equations [33]. In section 3, we present the procedure used to generate the POD basis. The main results is provided in section 4 where we prove a new error estimates between the standard finite elements Galerkin approximation of the solution and a spacial Galerkin approximation of the POD solution for the bidomain model. Finally, in section 5 we show how the POD reduced order method could be combined with time splitting schemes allowing to reduce the computational cost of the linear problem. Then, we carry out some numerical simulations showing the robustness of the POD method. The main conclusions of the study are then summarized in section 7 .

\section{Modelling and numerical methods}

\subsection{Electric model and some preliminary results}

The heart is assumed to be isolated, the propagation of the electrical wave in myocardium is governed by the following system of equations [33, 29, 8]:

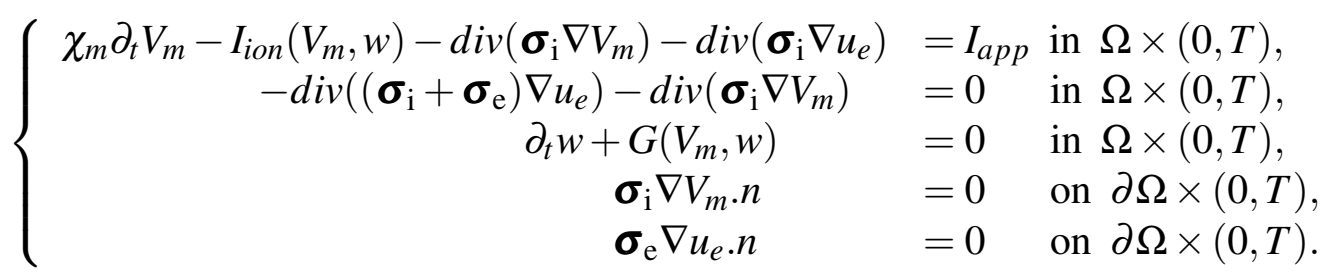


where $\Omega$ and $\partial \Omega$ denote respectively the heart domain and its boundary. The time domain is given by $(0, T)$, and $\chi_{m}$ the membrane capacitance per area unit. The variables $V_{m}$ and $u_{e}$ denote the action potential and the extracellular potential. The conductivity tensors $\boldsymbol{\sigma}_{\mathrm{i}}(x)$ in the intracellular domain and $\boldsymbol{\sigma}_{\mathrm{e}}(x)$ in the extracellular domain could then be written as follows

$$
\boldsymbol{\sigma}_{\mathrm{i}, \mathrm{e}}(x) \stackrel{\text { def }}{=} \sigma_{\mathrm{i}, \mathrm{e}}^{\mathrm{t}} \boldsymbol{I}+\left(\sigma_{\mathrm{i}, \mathrm{e}}^{1}-\sigma_{\mathrm{i}, \mathrm{e}}^{\mathrm{t}}\right) \boldsymbol{a}(x) \otimes \boldsymbol{a}(x),
$$

where $\boldsymbol{a}(x)$ is the fiber direction, $\boldsymbol{I}$ is the identity tensor and $\sigma_{\mathrm{i}, \mathrm{e}}^{1}$ and $\sigma_{\mathrm{i}, \mathrm{e}}^{\mathrm{t}}$ are conductivities values in the intra-cellular and extracellular along the fibers and in the transverse directions.

The term $I_{a p p}$ is a given source function, used in particular to initiate the activation, $w$ represents the concentrations of different chemical species, and variables representing the openings or closures of some gates of the ionic channels. The ionic current $I_{i o n}$ and the function $G\left(V_{m}, w\right)$ depends on the considered ionic model.

In [30], more than 28 models of cardiac cells are reported, some of them including more than 50 parameters [31]. In this study, the dynamics of $w$ and $I_{\text {ion }}$ are described by the Mitchell and Schaeffer model [22]:

$$
I_{\text {ion }}(v, w)=\frac{w}{\tau_{\text {in }}} v^{2}(1-v)-\frac{v}{\tau_{\text {out }}} \quad \text { and } \quad G(v, w)= \begin{cases}\frac{w-1}{\tau_{\text {open }}} & \text { if } v \leq v_{\text {gate }} \\ \frac{w}{\tau_{\text {close }}} & \text { if } v>v_{\text {gate }}\end{cases}
$$

The time constants $\tau_{\text {in }}, \tau_{\text {out }}$ are respectively related to the length of the depolarization and repolarization, $\tau_{\text {open }}$ and $\tau_{\text {close }}$ are the characteristic times of gate opening and closing respectively and $v_{\text {gate }}$ corresponds to the change-over voltage.

Problem (1) is completed with initial conditions

$$
V_{m}(0, x)=v_{0}(x) \quad \text { and } \quad w(0, x)=w_{0}(x) \quad \forall x \in \Omega .
$$

Finally, let us notice that $u_{e}$ is unique up to additive constant. This constant can be fixed by the following condition

$$
\int_{\Omega} u_{e} d x=0
$$

Let $[0, T]$ be a bounded interval of $\mathbb{R}$, we consider the Lebesgue measure $d t$ over the time interval $[0, T]$. We denote by $L^{p}(\Omega)$ the space of real functions which 
are in the $p$ th power integrable $(1 \leq p<\infty)$, or are measurable and essentially bounded (for $p=\infty$ ), and by $W^{1, p}(\Omega)$ the Sobolev space of functions $\psi: \Omega \rightarrow$ $\mathbb{R}$ which, together with their first-order weak partial derivatives, belong to the space $L^{p}(\Omega)(1 \leq p<\infty)$. For $X$ a Banach space, denote $C(0, T ; X)$ the space of continuous functions from $[0, T]$ into $X$ equipped with the uniform convergence norm, and

$$
\|f\|_{L^{p}(0, T ; X)}=\left(\int_{0}^{T}\|f(t)\|_{X}^{p} d t\right)^{1 / p} \quad\left(=\sup _{0<t<T} e s s\|f(t)\|^{p} \quad \text { if } \quad p=\infty\right) .
$$

By definition $L^{p}(0, T ; X), p<\infty$, is the seperated space of $C(0, T ; X)$ for this norm; for $p=\infty, L^{\infty}(0, T ; X)$ is the subset of $L^{1}(0, T ; X)$ on which the $L^{\infty}$ norm is finite. It is a Banach space for $0 \leq p \leq \infty$. For $p=2$ and $X=W^{1,2}(\Omega)$, we say space of Bochner integrable mappings, see [19]. Further, we set (see [6])

$$
W^{1,2}(\Omega) /_{\mathbb{R}}=\left\{u \in W^{1,2}(\Omega), \quad \int_{\Omega} u d x=0\right\} \subset W^{1,2}(\Omega),
$$

that is a Banach space with the norm $\|u\|_{W^{1,2}(\Omega) / \mathbb{R}}=\|u\|_{W^{1,2}(\Omega)}$. We have the Poincaré-Wirtinger inequality

$$
\exists C>0, \quad \forall u \in W^{1,2}(\Omega) / \mathbb{R}, \quad \int_{\Omega}|u|^{2} d x \leq C \int_{\Omega}|\nabla u|^{2} d x .
$$

The gradient $\nabla$ is always taken only with respect to the spatial variables $x$.

Let us make the following assumptions on the "data".

Assumption 1. Let $\Omega \in \mathbb{R}^{3}$ be a bounded domain with Lipschitz-continuous boundary $\partial \Omega$ and $0<T<\infty$. Furthermore define $Q:=\Omega \times[0, T]$ and $\Sigma=\partial \Omega \times[0, T]$.

Assumption 2. We assume that the conductivities of the intracellular and extracellular $\boldsymbol{\sigma}_{\mathrm{i}}, \boldsymbol{\sigma}_{\mathrm{e}} \in\left[L^{\infty}(\Omega)\right]^{3 \times 3}$ are symmetric and uniformly positive definite, i.e. there exist $\alpha_{i}>0$ and $\alpha_{e}>0$ such that, $\forall \xi \in \mathbb{R}^{3}$,

$$
\xi^{T} \boldsymbol{\sigma}_{\mathrm{i}}(x) \xi \geq \alpha_{i}|\xi|^{2}, \quad \xi^{T} \boldsymbol{\sigma}_{\mathrm{e}}(x) \xi \geq \alpha_{e}|\xi|^{2} .
$$

Namely, we assume that there exists constants $0<v<\mu$ such that

$$
v|\xi|^{2} \leq \xi^{T} \boldsymbol{\sigma}_{\mathrm{i}, \mathrm{e}} \xi \leq \mu|\xi|^{2} .
$$

Assumption 3. We assume that the function $I_{i o n}$ is Lipschitz with respect to the variable $V_{m}$. 
We have the following existence and regularity results of the weak solution of the bidomain system (1) (see [6, 24, 18, 38]).

Theorem 1. Let $T>0, I_{\text {app }} \in L^{2}\left(0, T ; W^{1,2}(\Omega)^{*}\right)$ and $v_{0} \in L^{2}(\Omega)$ be given data. If the assumptions (1)-(3) hold and $w_{0} \in L^{2}(\Omega)$, such that

$$
0 \leq w_{0} \leq 1 \quad \text { in } \Omega,
$$

then, system (1) has a weak solution in the following sense:

$$
\begin{aligned}
& V_{m} \in C\left(0, T ; L^{2}(\Omega)\right) \cap L^{2}\left(0, T ; W^{1,2}(\Omega)\right) \cap L^{4}(Q) \\
& u_{e} \in L^{2}\left(0, T ; L^{2}(\Omega)\right) \text { and } \quad w \in C\left(0, T ; L^{2}(\Omega)\right),
\end{aligned}
$$

such that for all $\psi_{1} \in W^{1,2}(\Omega)$ and $\psi_{2} \in W^{1,2}(\Omega) / \mathbb{R}$

$$
\begin{gathered}
\chi_{m} \int_{\Omega} \partial_{t} V_{m} \psi_{1} d x+\int_{\Omega} \boldsymbol{\sigma}_{\mathrm{i}} \nabla V_{m} \nabla \psi_{1} d x+\int_{\Omega} \boldsymbol{\sigma}_{\mathrm{i}} \nabla u_{e} \nabla \psi_{1} d x \\
+\int_{\Omega} I_{i o n}\left(V_{m}, w\right) \psi_{1} d x=\int_{\Omega} I_{a p p} \psi_{1} d x, \\
\int_{\Omega} \boldsymbol{\sigma}_{\mathrm{i}} \nabla V_{m} \nabla \psi_{2} d x+\int_{\Omega}\left(\boldsymbol{\sigma}_{\mathrm{i}}+\boldsymbol{\sigma}_{\mathrm{e}}\right) \nabla u_{e} \nabla \psi_{2} d x=0, \\
\partial_{t} w+G\left(V_{m}, w\right)=0 .
\end{gathered}
$$

Moreover, $0 \leq w \leq 1$ a.e. in $Q$, and there exists a constant $C>0$ such that the following a-priori bound holds:

$$
\begin{aligned}
\left\|V_{m}\right\|_{L^{2}\left(0, T ; W^{1,2}(\Omega)\right)}^{2}+\left\|V_{m}\right\|_{L^{4}(Q)}^{2} & +\left\|u_{e}\right\|_{L^{2}\left(0, T ; L^{2}(\Omega)\right)}^{2} \leq C\left(1+\left\|v_{0}\right\|_{L^{2}(\Omega)}^{2}\right. \\
& \left.+\left\|w_{0}\right\|_{L^{2}(\Omega)}^{2}+\left\|I_{a p p}\right\|_{L^{2}\left(0, T ; W^{1,2}(\Omega)^{*}\right)}^{2}\right) .
\end{aligned}
$$

\subsection{Abstract discretization of bidomain system}

In order to actually compute a solution of system(1), we shall discretize the problem and we try to approximate the problem in a finite dimension subspace. We approximate $W^{1,2}(\Omega)$ by a finite dimensional space, spanned by "Galerkin functions" $\left(\varphi_{k}\right)_{k=1, \cdots, M}$ :

$$
W_{h}:=\operatorname{span}\left(\varphi_{1}, \cdots, \varphi_{M}\right) \subset W^{1,2}(\Omega), \quad M \in \mathbb{N} .
$$

A suitable initial value $v_{0, h}$ may be obtained by an $(., .)_{W^{1,2}(\Omega)^{-}}$-orthogonal projection of $v_{0} \in W^{1,2}(\Omega)$ on $W_{h}$, for instance. The discrete problem statement then reads: 
Problem 1. Find $\left(V_{m, h}, u_{e, h}\right) \in L^{2}\left(0, T ; W_{h}\right) \times L^{2}\left(0, T ; W_{h}\right) / \mathbb{R}$ where we have $\nabla V_{m, h} \in L^{2}\left(0, T ; W_{h}^{*}\right)$, such that for an appropriate initial value $v_{0, h} \in W^{1,2}(\Omega)$, there holds

$$
\begin{aligned}
& \chi_{m} \int_{\Omega} \partial_{t} V_{m, h} \psi_{1} d x+\int_{\Omega} \boldsymbol{\sigma}_{\mathrm{i}} \nabla V_{m, h} \nabla \psi_{1} d x \\
&+\int_{\Omega} \boldsymbol{\sigma}_{\mathrm{i}} \nabla u_{e, h} \nabla \psi_{1} d x=\int_{\Omega}\left(I_{\text {app }}-I_{\text {ion }}\left(V_{m, h}, w_{h}\right)\right) \psi_{1}, \forall \psi_{1} \in W_{h}, \\
& \int_{\Omega} \boldsymbol{\sigma}_{\mathrm{i}} \nabla V_{m, h} \nabla \psi_{2} d x+\int_{\Omega}\left(\boldsymbol{\sigma}_{\mathrm{i}}+\boldsymbol{\sigma}_{\mathrm{e}}\right) \nabla u_{e, h} \nabla \psi_{2} d x=0 \quad \forall \psi_{2} \in W_{h} / \mathbb{R} \\
& V_{m, h}(0, .)=v_{0, h} \in W^{1,2}(\Omega) \text { and } u_{e, h}(0, .)=u_{e, 0, h} \in L^{2}(\Omega) .
\end{aligned}
$$

Due to the non-linearity of the reaction term most of works use a semi-implicit scheme for the time discretization to solve the bidomain equations [38, 35, 8, 10, $21,4,34,14,27]$, problem (1) is equivalent to a linear system whose matrix in $\mathbb{R}^{2 M \times 2 M}$ and given by

$$
\left\{\begin{aligned}
\left(\frac{\chi_{m}}{\delta t} S_{1}+S_{2}\right) \mathbf{V}_{m}^{n+1}+S_{2} \mathbf{u}_{e}^{n+1} & =\frac{\chi_{m}}{\delta t} S_{1} \mathbf{V}_{m}^{n}+M\left(I_{\text {app }}^{n+1}-I_{\text {ion }}\left(\mathbf{V}_{m}^{n}, w^{n+1}\right)\right) \\
S_{2} \mathbf{V}_{m}^{n+1}+S_{3} \mathbf{u}_{e}^{n+1} & =0 \\
\mathbf{w}^{n+1} & =\mathbf{w}^{n}-\delta t G\left(\mathbf{V}_{m}^{n}, w^{n+1}\right)
\end{aligned}\right.
$$

where $\mathbf{V}_{m}^{n}, \mathbf{u}_{e}^{n}$ and $\mathbf{w}^{n} \in \mathbb{R}^{M}$ are the vectors the contain the value of $V_{m, h}, u_{e, h}$ and $w_{h}$ at time $t_{n}$

$$
\left(\begin{array}{ll}
\frac{\chi_{m}}{\delta t} S_{1}+S_{2} & S_{2} \\
S_{2} & S_{3}
\end{array}\right) \times\left(\begin{array}{l}
\mathbf{V}_{m}^{n+1} \\
\mathbf{u}_{e}^{n+1}
\end{array}\right)=\left(\begin{array}{l}
\frac{\chi_{m}}{\delta t} S_{1} \mathbf{V}_{m}^{n}+S_{1}\left(I_{a p p}^{n+1}-I_{i o n}\left(\mathbf{V}_{m}^{n}, w^{n+1}\right)\right) \\
0
\end{array}\right)
$$

where matrices $S_{1} \in \mathbb{R}^{M \times M}, S_{2} \in \mathbb{R}^{M \times M}$ and $S_{3} \in \mathbb{R}^{M \times M}$ are defined by

$$
\begin{gathered}
S_{1}=\left(\int_{\Omega} \varphi_{i} \varphi_{j}\right)_{i, j=1, \cdots, M} \quad S_{2}=\left(\int_{\Omega} \sigma_{\mathrm{i}} \nabla \varphi_{i} \nabla \varphi_{j}\right)_{i, j=1, \cdots, M} \\
S_{3}=\left(\int_{\Omega}\left(\sigma_{\mathrm{i}}+\boldsymbol{\sigma}_{\mathrm{e}}\right) \nabla \varphi_{i} \nabla \varphi_{j}\right)_{i, j=1, \cdots, M}
\end{gathered}
$$


Remark 1. The subset $W_{h}=\operatorname{span}\left(\varphi_{1}, \cdots, \varphi_{M}\right)$ is in general an approximation of $W^{1,2}(\Omega)$. This approximation could be done using the standard finite element basis for example $\left(e_{1}, \cdots, e_{M}\right)$. If we consider a first order approximation (known as P1), the basis elements are piecewise polynomial functions. The size of the linear system to solve in this case is $2 \times M$, where $M$ represents also the number of nodes in the space discretization of the domain $\Omega$.

The goal of this work is to reduce the size of the linear system to solve, by projecting the variational formulation on a very low dimensional subspace of the finite element $\operatorname{space} \operatorname{span}\left(e_{1}, \cdots, e_{M}\right)$ and to demonstrate that this approach is stable.

\section{Reduced order method}

A full order code based on the finite elements method requires many thousands of degrees of freedom for the accurate numerical simulation of cardiac electrophysiology. To reduce its complexity, following [4, 2], we choose to use the POD method (see [16, 37]). This method is applied to data compression and model reduction of finite dimensional nonlinear systems, it's still known in other areas of science as the Karhunen-Loève (Karhunen, 1946; Loève, 1955). Today, POD analysis is the most popular reduced-order modeling approach for complex systems, the first natural question to ask is: How does one determine a reduced basis of very low dimension? In the following section, we briefly recall the principle of the POD method.

\subsection{Generation of the reduced order basis}

For a given dimension $K \leq N_{t}$, our goal is to determine a POD basis $\left(\phi_{k}\right)_{k=1}^{K}$ of rank $K$ such that describes best a "snapshot set":

$V_{P}:=\left\{V_{m, k}=V_{m}\left(t_{k},.\right) \mid t_{k} \in \Gamma_{N_{t}}\right\} \subset \mathbb{R}^{2 M} \quad$ with $\quad \Gamma_{N_{t}}:=\left\{t_{k} \in[0, T] \mid k=1, \cdots N_{t}\right\}$,

In this section we detail the case of an explicit method. The implicit one is similar. We look for a reduced order basis $\Phi=\left(\phi_{k}\right)_{k=1}^{K}$ that is able to accurately 
regenerate the data stored in the following snapshots matrix

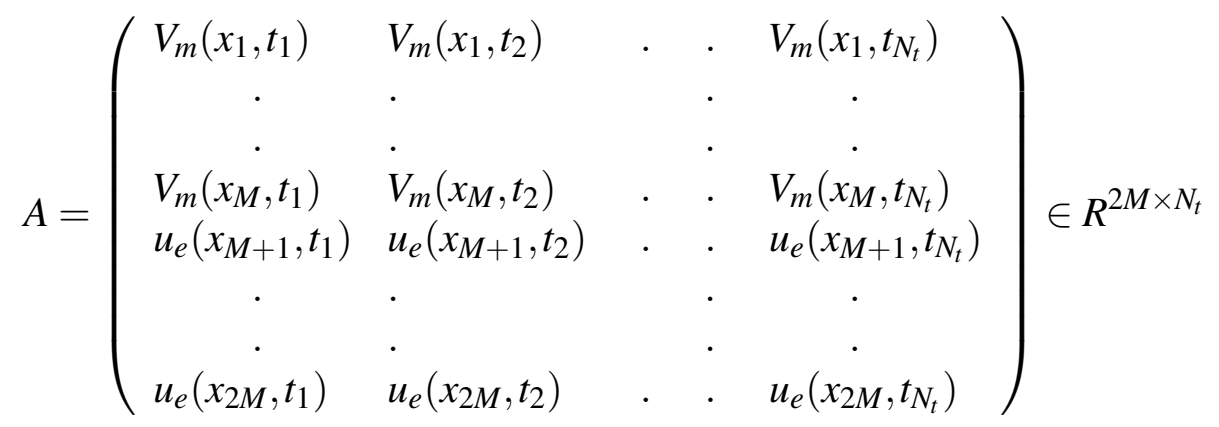

In our case, the snapshots matrix contains a solution precomputed using a full order method based on the finite elements method. The K-dimensional POD basis is then determined from the $\mathrm{K}$ eigenvectors corresponding to the $\mathrm{K}$ most dominant eigenvalues of the correlation matrix for the snapshots. Then we determine the orthonormal basis $\phi_{k}(x)_{k=1}^{K}$ with $K<N_{t}$ solution of the problem:

$$
\min _{\left(\phi_{k}\right)_{k=1}^{K} \in R^{2 M}} \sum_{i=1}^{N_{t}}\left\|u\left(x, t_{i}\right)-\sum_{k=1}^{K}\left(u\left(x, t_{i}\right), \phi_{k}(x)\right) \phi_{k}(x)\right\|_{\text {euclidean norm }}^{2}
$$

where

$u\left(x, t_{i}\right)=\left(V_{m}\left(x_{1}, t_{i}\right), \cdots, \quad V_{m}\left(x_{M}, t_{i}\right), \quad u_{e}\left(x_{1}, t_{i}\right), \cdots, \quad u_{e}\left(x_{M}, t_{i}\right)\right)^{T} \in \mathbb{R}^{2 M \times 1}$

This minimization problem is solved using the singular value decomposition (SVD) procedure. See $[28,36]$ for more details. There are three ways of computing the basis $\left(\phi_{k}\right)_{k=1}^{K}$ :

- Calculate a SVD of $A=U D V^{T}$ and let $\left\{\phi_{k}\right\}_{k=1}^{K}$ consist of the first $K$ columns of $U$.

- (Classical POD) Find "orthonormal" eignvectors to the $K$ largest eigenvalues of:

$$
A A^{T} \phi_{k}=\lambda_{k} \phi_{k} \quad \text { in } \quad \mathbb{R}^{2 M}
$$

- Find "orthonormal" eigenvectors to the $K$ largest eigenvalues of

$$
A^{T} A u_{k}=\lambda_{k} u_{k} .
$$

Then, the POD basis of rank $K$ for this case, is given by $\left\{\phi_{k}\right\}_{k=1}^{K}$ which consists of

$$
\phi_{k}:=\frac{1}{\sqrt{\lambda_{k}}} A u_{k}, \quad k=1, \cdots, K .
$$


Remark 2. For large scale problems, the SVD method is not a good choice in terms of computational cost and machine memory issues. Since we are only interested in computing the $K$ first columns of $U$, in practice, we use the second or the third methods depending on the size of $A A^{T}$ and $A A^{T}$.

\subsection{Solving the reduced order problem}

Instead of being projected on the full finite element basis, the variational formulation (12) would now be projected on the POD basis $\Phi=\left(\phi_{k}\right)_{k=1}^{K}$. Knowing the reduced order solution $\left(V_{p o d}^{n} ; u_{e, p o d}^{n}\right)=\sum_{k=1}^{K} \mathbf{u}_{\text {pod,k }}^{n} \phi_{k}$ at time $t_{n}$, we look for the reduced order solution $\left(V_{\text {pod }}^{n+1}, u_{e, p o d}^{n+1}\right)=\sum_{k=1}^{K} \mathbf{u}_{\text {pod,k }}^{n+1} \phi_{k}$ where $\mathbf{u}_{\text {pod }}^{n+1}=\left(\mathbf{u}_{\text {pod, }, k}^{n+1}\right)_{k=1}^{K}$ is solution of the following reduced order problem

$\Phi^{T}\left(\begin{array}{ll}\chi_{m} S_{1}+\delta t S_{2} & \delta t S_{2} \\ S_{2} & S_{3}\end{array}\right) \Phi \mathbf{u}_{\text {pod }}^{n+1}=\Phi^{T}\left(\begin{array}{l}S_{1}\left(\chi_{m} V_{p o d}^{n}+\delta t\left(I_{\text {app }}^{n+1}-I_{\text {ion }}\left(V_{p o d}^{n}, w^{n+1}\right)\right)\right) \\ 0\end{array}\right)$

Remark 3. The left hand side matrix is the reduced order matrix and could be easily inverted once for all in our problem. The right hand side is the finite element right hand-side vector projected on the POD basis and it has to be updated at each time step.

\section{New estimates for the POD reduction error}

Our aim is to control the gap between the reduced order and the finite element bidomain solutions. The problem (1) could be rewritten as a single abstract parabolic equation with the action potential $V_{m}$ and w as unknowns, see for example [6]

$$
\begin{cases}\chi_{m} \partial_{t} V_{m}+A V_{m}=I_{a p p}-I_{i o n}\left(V_{m}, w\right) & \text { in } \Omega \times(0, T) \\ \partial_{t} w+G\left(V_{m}, w\right)=0 & \text { in } \Omega \times(0, T) \\ \boldsymbol{\sigma}_{\mathrm{i}} \nabla V_{m} \cdot n=0 & \text { on } \Sigma\end{cases}
$$

where $A$ is an abstract integro-differential second order elliptic operator given by

$$
A u=-\operatorname{div}\left(\boldsymbol{\sigma}_{\mathrm{i}} \nabla u\right)+\operatorname{div}\left(\boldsymbol{\sigma}_{\mathrm{i}} \nabla\left\{\operatorname{div}\left(\boldsymbol{\sigma}_{\mathrm{i}}+\boldsymbol{\sigma}_{\mathrm{e}}\right) \nabla\right\}^{-1}\left(\operatorname{div}\left(\boldsymbol{\sigma}_{\mathrm{i}} \nabla u\right)\right)\right.
$$

This representation has been used in order to show the existence of a solution for the bidomain problem [6]. In the Galerkin approach for Problem (15), we choose $V_{h}$ to be $\Phi=\operatorname{span}\left\{\phi_{1}, \cdots, \phi_{K}\right\}$ and denote the corresponding solution by $V_{m}^{K}$ instead of $V_{m, h}$. Then we have,

$$
V_{m, h} \in \Phi,
$$




$$
\begin{gathered}
\chi_{m} \int_{\Omega} \partial_{t} V_{m}^{K} \psi d x+\int_{\Omega} A V_{m}^{K} \psi d x=\int_{\Omega}\left(I_{a p p}-I_{i o n}\left(V_{m}^{K}, w_{h}\right)\right) \psi d x \quad \forall \psi \in \Phi \\
V_{m}^{K}(0, .)=v_{0}^{K} .
\end{gathered}
$$

We refer to [7], that if $I_{\text {app }}-I_{\text {ion }} \in L^{2}\left(0, T ; L^{2}(\Omega)\right)$ and $v_{0}^{K} \in \Phi$, then there exists a unique solution $V_{m}^{K}$ of Eqs. (18)-(19) such that $V_{m}^{K} \in C([0, T] ; \Phi)$.

Let $P_{L^{2}}^{K}$ the $L^{2}(\Omega)$-orthogonal projectors onto the reduction space $\Phi$. For all $v \in W^{1,2}(\Omega)$

$$
\left\|v-P_{L^{2}}^{K} v\right\|_{L^{2}(\Omega)}=\inf _{v^{K} \in \Phi}\left\|v-v^{K}\right\|_{L^{2}(\Omega)} .
$$

For $u, v \in W^{1,2}(\Omega)$ we write

$$
(u, v):=\int_{\Omega} u v d x, \quad a(u, v):=\frac{1}{\chi_{m}} \int_{\Omega} A u v d x
$$

$$
\text { and } f:[0, T] \times W^{1,2}(\Omega) \rightarrow W^{1,2}(\Omega),(t, u) \mapsto \frac{1}{\chi_{m}}\left(I_{\text {app }}(t)-I_{\text {ion }}(u, w)\right)
$$

The function $f$ is Lipschitz because of the assumption (Assumption 3).

We decompose the POD Galerkin error expression as follows

$$
V_{m}-V_{m, p o d}=p^{K}+q^{K}
$$

into the time discretization error

$$
p^{K}:=V_{m}-P_{L^{2}}^{K} V_{m}
$$

as well as the restriction to the POD subspace error

$$
q^{K}:=P_{L^{2}}^{K} V_{m}-V_{m, p o d}
$$

where we denote by $V_{m \text {,pod }}$ the spacial Galerkin approximation in the POD space $\Phi=\operatorname{span}\left\{\varphi_{1}, \cdots, \varphi_{K}\right\}$ and by $Q=L^{2}\left(0, T ; W^{1,2}(\Omega)\right)$. Our main result in this section is the following:

Theorem 2. For all $T \geq 0$, if $\left(V_{m}, u_{e}\right)$ is the solution of the bidomain equations (12) computed with the full order method based in the finite elements method and $\left(V_{m, p o d}, u_{e, p o d}\right)$ is a spatial Galerkin approximation in the POD space $\Phi=$ $\operatorname{span}\left\{\phi_{1}, . ., \phi_{K}\right\}$, then, there exists three constants $C_{1}, C_{2}, C_{3}>0$ such that 


$$
\begin{aligned}
& \left\|V_{m}-V_{m, p o d}\right\|_{Q} \leq C_{1}\left(\left\|P_{L^{2}}^{K} v_{0}-v_{0, p o d}\right\|_{L^{2}(\Omega)}+\left\|V_{m}-P_{L^{2}}^{K} V_{m}\right\|_{Q}\right) \\
& \left\|u_{e}-u_{e, p o d}\right\|_{Q} \leq C_{2}\left\|V_{m, p o d}-V_{m}\right\|_{Q} .
\end{aligned}
$$

Or equivalently,

$$
\begin{gathered}
\left\|V_{m}-V_{m, p o d}\right\|_{Q} \leq C_{1}\left(\left\|P_{L^{2}}^{K} v_{0}-v_{0, p o d}\right\|_{L^{2}(\Omega)}+\left\|V_{m}-P_{L^{2}}^{K} V_{m}\right\|_{Q}\right) \\
\left\|u_{e}-u_{e, p o d}\right\|_{Q} \leq C_{3}\left(\left\|P_{L^{2}}^{K} u_{e, 0}-u_{e, 0, p o d}\right\|_{L^{2}(\Omega)}+\left\|u_{e}-P_{L^{2}}^{K} u_{e}\right\|_{Q}\right)
\end{gathered}
$$

Remark 4. The first estimate (21)-(22) shows that if the projection of the finite element solution on the POD basis is able to sufficiently represent the dynamic of the action potential $V_{m}$, we control the error between the finite element solution and the POD solution for both state variables $V_{m}$ and $u_{e}$. The second estimate (23)-(24) shows that the error between the finite element and the POD solutions for $V_{m}$ (respectively, $u_{e}$ ) is proportional to the the error between $V_{m}$ (respectively, $u_{e}$ ) and its projection on the POD basis $P_{L^{2}}^{K} V_{m}$, (respectively, $P_{L^{2}}^{K} u_{e}$ ).

To prove Theorem 2, we would need a preliminary estimate. The following lemma establishes an a priori estimate on $q^{K}$.

Lemma 3. Under the Assumption (2) and Assumption (3) we have

$$
\left\|q^{K}\right\|_{Q}^{2} \leq \frac{e^{2 L T}}{c_{a}}\left(\left\|q^{K}(0)\right\|_{L^{2}(\Omega)}^{2}+\frac{\left(C_{a}+L C_{\Omega}^{2}\right)^{2}}{c_{a}}\left\|p^{K}\right\|_{Q}\right),
$$

where $c_{a}=\frac{1}{\chi_{m}} \min \left(\alpha / 2, \alpha / 2 c_{p}\right)$ given by the coercivity of the bilinear form $a$, $C_{a}=\frac{3 \mu}{\chi_{m}}(1+M \mu / 2 v)$ is the continuity constant of $a$, with $\alpha=\frac{v}{3}(1+v / 2 \mu)$ and $c_{p}$ is the constant of the Poincaré inequality.

Proof. The solution $V_{m, p o d}$ verify, for all $v \in \Phi$

$$
\left(\partial_{t} V_{m, p o d}, v\right)+a\left(V_{m, p o d}, v\right)=\left(f\left(V_{m, p o d}\right), v\right)
$$

Taking $v=q^{K}$ and $V_{m, p o d}=P_{L^{2}}^{K} V_{m}-q^{K}$, we can write $\left(\partial_{t} q^{K}(t), q^{K}(t)\right)+a\left(q^{K}(t), q^{K}(t)\right)=-a\left(p^{K}(t), q^{K}(t)\right)+\left(f\left(V_{m}\right)-f\left(V_{m, p o d}\right), q^{K}(t)\right)$. 
We integrate on $[0, t]$ for $0 \leq t \leq T$ and using the coercivity of the bilinear form $a$ (see [6]), we obtain the following estimation:

$$
\begin{aligned}
\frac{1}{2}\left\|q^{K}(t)\right\|_{L^{2}(\Omega)}^{2}+ & c_{a}\left\|q^{K}\right\|_{L^{2}\left(0, t ; W^{1,2}(\Omega)\right)}^{2} \leq \frac{1}{2}\left\|q^{K}(0)\right\|_{L^{2}(\Omega)}^{2}+L\left\|q^{K}\right\|_{L^{2}\left(0, t ; L^{2}(\Omega)\right)}^{2} \\
& +\left(C_{a}+L C_{\Omega}^{2}\right)\left\|p^{K}\right\|_{L^{2}\left(0, t ; W^{1,2}(\Omega)\right)}\left\|q^{K}\right\|_{L^{2}\left(0, t ; W^{1,2}(\Omega)\right)}
\end{aligned}
$$

where the constant $C_{\Omega}$ verify $\|v\|_{L^{2}(\Omega)} \leq C_{\Omega}\|v\|_{W^{1,2}(\Omega)} \forall v \in W^{1,2}(\Omega)$.

Applying the young inequality

$$
x y \leq \frac{x^{p}}{\varepsilon p}+\frac{\varepsilon y^{q}}{q}
$$

for $x=\left(C_{a}+L C_{\Omega}^{2}\right)\left\|p^{k}\right\|_{L^{2}\left(0, T ; W^{1,2}(\Omega)\right)}, y=\left\|q^{k}\right\|_{L^{2}\left(0, t ; W^{1,2}(\Omega)\right)}, p=q=2$ and $\varepsilon=c_{a}$, we can deduce

$$
\begin{aligned}
\left\|q^{K}(t)\right\|_{L^{2}(\Omega)}^{2}+c_{a}\left\|q^{K}\right\|_{L^{2}\left(0, t ; W^{1,2}(\Omega)\right)}^{2} & \leq\left\|q^{K}(0)\right\|_{L^{2}(\Omega)}^{2}+2 L\left\|q^{K}\right\|_{L^{2}\left(0, t ; L^{2}(\Omega)\right)}^{2} \\
& +\frac{\left(C_{a}+L C_{\Omega}^{2}\right)^{2}}{c_{a}}\left\|p^{K}\right\|_{\Omega}^{2}
\end{aligned}
$$

From the Gronwall's inequality for $t \mapsto\left\|q^{K}(t)\right\|_{L^{2}(\Omega)}^{2}$, we have

$$
\left\|q^{K}(t)\right\|_{L^{2}(\Omega)}^{2} \leq e^{2 L t}\left(\left\|q^{K}(0)\right\|_{L^{2}(\Omega)}^{2}+\frac{\left(C_{a}+L C_{\Omega}^{2}\right)^{2}}{c_{a}}\left\|p^{K}\right\|_{\Omega}^{2}\right)
$$

We integrate on $[0, t]$, we obtain:

$$
2 L\left\|q^{K}\right\|_{L^{2}\left(0, t ; L^{2}(\Omega)\right)}^{2} \leq\left(e^{2 L t}-1\right)\left(\left\|q^{K}(0)\right\|_{L^{2}(\Omega)}^{2}+\frac{\left(C_{a}+L C_{\Omega}^{2}\right)^{2}}{c_{a}}\left\|p^{K}\right\|_{Q}^{2}\right)
$$

From (26)-(27)-(29), we have

$$
\begin{aligned}
c_{a}\left\|q^{K}\right\|_{L^{2}\left(0, t ; W^{1,2}(\Omega)\right)}^{2} & \leq\left\|q^{K}(t)\right\|_{L^{2}(\Omega)}^{2}+c_{a}\left\|q^{K}\right\|_{L^{2}\left(0, t ; W^{1,2}(\Omega)\right)}^{2} \\
& \leq\left\|q^{K}(0)\right\|_{L^{2}(\Omega)}^{2}+2 L\left\|q^{K}\right\|_{L^{2}\left(0, t ; L^{2}(\Omega)\right)}^{2} \\
& +\frac{\left(C_{a}+L C_{\Omega}^{2}\right)^{2}}{c_{a}}\left\|p^{K}\right\|_{L^{2}\left(0, t ; W^{1,2}(\Omega)\right)}^{2} \\
& \leq e^{2 L t}\left(\left\|q^{K}(0)\right\|_{L^{2}(\Omega)}^{2}+\frac{\left(C_{a}+L C_{\Omega}^{2}\right)^{2}}{c_{a}}\left\|p^{K}\right\|_{Q}^{2}\right),
\end{aligned}
$$


Let us now complete the proof of Theorem 2.

Proof of TheOREM 2. For the first inequality, using the decomposition (20) and Lemma 3, we have

$$
\begin{aligned}
\left\|V_{m}-V_{m, p o d}\right\|_{Q} & \leq\left\|p^{K}\right\|_{Q}+\left\|q^{K}\right\|_{Q} \\
& \leq\left\|p^{K}\right\|_{Q}+\frac{e^{L T}}{\sqrt{c_{a}}}\left(\left\|q^{K}(0)\right\|_{L^{2}(\Omega)}+\frac{C_{a}+L C_{\Omega}^{2}}{\sqrt{c_{a}}}\left\|p^{K}\right\|_{Q}\right) \\
& \leq C_{1}\left(\left\|P_{L^{2}}^{K} v_{0}-v_{0, p o d}\right\|_{L^{2}(\Omega)}+\left\|V_{m}-P_{L^{2}}^{K} V_{m}\right\|_{Q}\right)
\end{aligned}
$$

with $C_{1}=\max \left(\frac{e^{L T}}{\sqrt{c_{a}}}, 1+\frac{C_{a}+L C_{\Omega}}{\sqrt{c_{a}}}\right)$.

Let us now prove the second estimation (22). In $\Omega \times[0, T]$ we have

$$
-\operatorname{div}\left(\left(\boldsymbol{\sigma}_{\mathrm{i}}+\boldsymbol{\sigma}_{\mathrm{e}}\right) \nabla u_{e}\right)-\operatorname{div}\left(\boldsymbol{\sigma}_{\mathrm{i}} \nabla V_{m}\right)=0
$$

and

$$
-\operatorname{div}\left(\left(\boldsymbol{\sigma}_{\mathrm{i}}+\boldsymbol{\sigma}_{\mathrm{e}}\right) \nabla u_{e, p o d}\right)-\operatorname{div}\left(\boldsymbol{\sigma}_{\mathrm{i}} \nabla V_{m, p o d}\right)=0
$$

We multiply by a test function $v \in W^{1,2}(\Omega)$ and integrate by parts, we obtain

$$
\int_{\Omega}\left(\boldsymbol{\sigma}_{\mathrm{i}}+\boldsymbol{\sigma}_{\mathrm{e}}\right) \nabla\left(u_{e}-u_{e, p o d}\right) \nabla v d x+\int_{\Omega} \boldsymbol{\sigma}_{\mathrm{i}} \nabla\left(V_{m}-V_{m, p o d}\right) \nabla v d x=0,
$$

taking $v=u_{e}-u_{e, p o d}$, we deduce that

$$
\int_{\Omega}\left(\boldsymbol{\sigma}_{\mathrm{i}}+\boldsymbol{\sigma}_{\mathrm{e}}\right) \nabla\left(u_{e}-u_{e, p o d}\right) \nabla\left(u_{e}-u_{e, p o d}\right)=\int_{\Omega} \boldsymbol{\sigma}_{\mathrm{i}} \nabla\left(V_{m, p o d}-V_{m}\right) \nabla\left(u_{e}-u_{e, p o d}\right) .
$$

Using the assumption (2) in the left hand side and the Cauchy-Schwarz inequality in the right hand side, we obtain

$$
\left\|\nabla\left(u_{e}-u_{e, p o d}\right)\right\|_{L^{2}(\Omega)} \leq \frac{\mu}{2 v}\left\|\nabla\left(V_{m, p o d}-V_{m}\right)\right\|_{L^{2}(\Omega)} .
$$

Using Poincaré-Wirtinger inequality (2) on the left hand side and by integrating on $[0, T]$, we conclude that

$$
\left\|u_{e}-u_{e, p o d}\right\|_{Q} \leq \frac{\mu C}{2 v}\left\|V_{m, p o d}-V_{m}\right\|_{Q} .
$$

This proves (22).

In order to prove (24), we use the following integro-differential equation

$$
\left.V_{m}=\left\{\operatorname{div}\left(\boldsymbol{\sigma}_{\mathrm{i}} \nabla\right)\right\}^{-1}\left(\operatorname{div}\left(\boldsymbol{\sigma}_{\mathrm{i}}+\boldsymbol{\sigma}_{\mathrm{e}}\right) \nabla u_{e}\right)\right):=B u_{e},
$$

We can see that the operator $B$ is linear and uniformly continuous. Replacing the new expression of $V_{m}$ in the right hand side of (22), using the estimate (21) and the uniform contunuity of $B$ we deduce the second estimate (24). 


\section{2D Numerical results}

In this section, we present numerical simulations about the POD approximation of the bidomain model. We use the Mitchell and Schaeffer model [22] to describe the ionic properties of the cell membrane. For the sake of simplicity and reproducibility, the heart domain is the square $\Omega=[0,1] \times[0,1]$ and the unit is $\mathrm{cm}$. It is discretized on the $\mathrm{x}$ and $\mathrm{y}$-axis with a space step $0.02 \mathrm{~cm}$. The time domain is $[0,500]$, its unit is $\mathrm{ms}$ and the time step is $0.2 \mathrm{~ms}$. The heart is stimulated in a region at the left bottom corner $[0,0.025] \times[0,0.025]$ during $2 \mathrm{~ms}$. The parameter of the ionic model are given in Table 1.

\begin{tabular}{lllll}
\hline$\tau_{\text {close }}$ & $\tau_{\text {open }}$ & $\tau_{\text {in }}$ & $\tau_{\text {out }}$ & $V_{\text {gate }}$ \\
150 & 120 & 0.3 & 6 & 0.13
\end{tabular}

Table 1: Original Mitchell and Schaeffer cell membrane parameters.

As mentioned in the previous section we start by generating a solution of the bidomain problem. All the time steps of the full finite element solution are stored in the snapshot matrix and used to construct the POD basis as explained in section 3.1. In this example we have 2601 degrees of freedom. Capturing $99.99 \%$ the finite element solution energy requires only 69 modes. We use these 69 modes as a basis and we solve the POD problem. Using a matlab code, the full finite element solution costs $0.47 \mathrm{~s}$, where the ODE system costs $0.0011 \mathrm{~s}$ and the linear system costs $0.47 \mathrm{~s}$. The solution of the reduced order problem is computed in $0.05 \mathrm{~s}$, where 0.0011 for the ODE system and $0.049 \mathrm{~s}$ for the linear system. In Figure 1, we show the snapshots of the full finite element solution (top) and the POD solution (bottom) at the depolarization phase in the isotropic case. We performed the same study for an anisotropic case where the longitudinal direction of the fibers is given by $\boldsymbol{a}(x)=\left(\frac{1}{\sqrt{2}}, \frac{1}{\sqrt{2}}\right)$ and the transverse direction is given by $\left(-\frac{1}{\sqrt{2}}, \frac{1}{\sqrt{2}}\right)$. Here we consider that the conductivity along the fibers is three times higher than it is in the transverse direction. In Figure 2 we show the snapshots of the full finite element solution (top) and the POD solution (bottom) at the depolarization phase for the anisotropic case. We see, in both cases, that there is no difference between the full finite element and the POD solution in terms pattern. The wave front of the electrical potential is accurately captured.

\subsection{Combining the POD method with time splitting schemes}

In order to reduce the computational cost when solving the bidomain equations some numerical schemes have been introduced in order to uncouple the compu- 
$V_{m}$

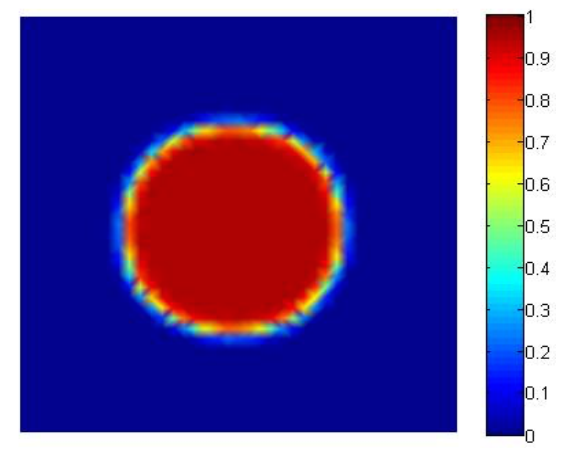

$V_{m, p o d}$

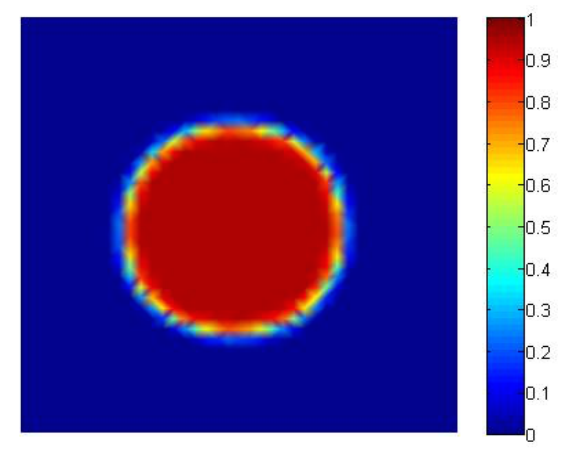

$u_{e}$

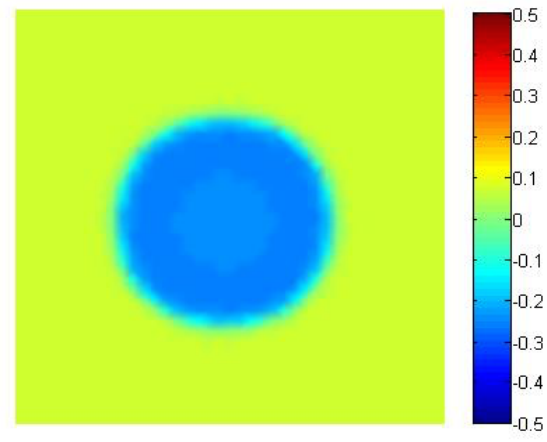

$u_{e, p o d}$

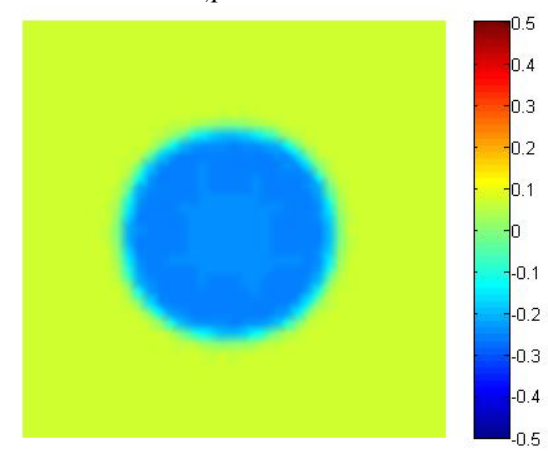

Figure 1: Top (respectively, bottom): Snapshots of the full finite element (respectively POD) solution at time $20 \mathrm{~ms}$. at the depolarization phase. The action potential distribution is in the left column and the extracellular potential distribution is in the right column.

tation of the action potential and the extracellular potential [29, 20, 1, 35]. The authors propose a decoupled (Gauss-Seidel-like) time-marching schemes allowing at each time step to solve first the action potential and then the extracellular potential. After performing time and space discretization, this scheme reads as follows:

- Solve the ionic model:

$$
w^{n+1}=w^{n}-\delta t G\left(V_{m}^{n}, w^{n+1}\right)
$$

- Solve the action potential :

$$
\left(\chi_{m} S_{1}+\delta t S_{2}\right) V_{m}^{n+1}=-\delta t S_{2} u_{e}^{n}+\chi_{m} S_{1} V_{m}^{n}+\delta t S_{1}\left(I_{a p p}^{n+1}-I_{i o n}\left(V_{m}^{n}, w^{n+1}\right)\right)
$$




$$
V_{m}
$$

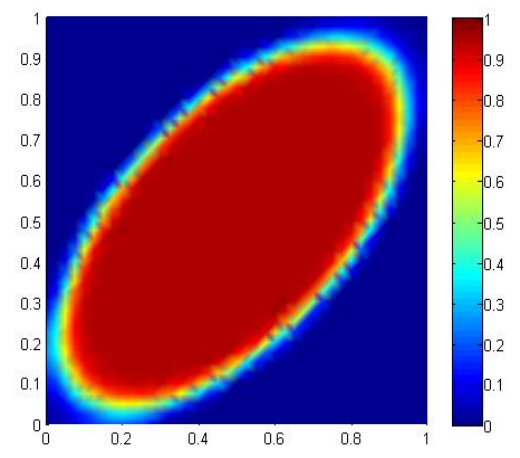

$$
V_{m, p o d}
$$

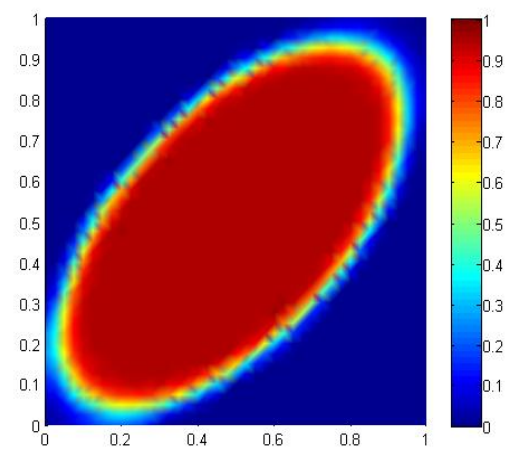

$u_{e}$

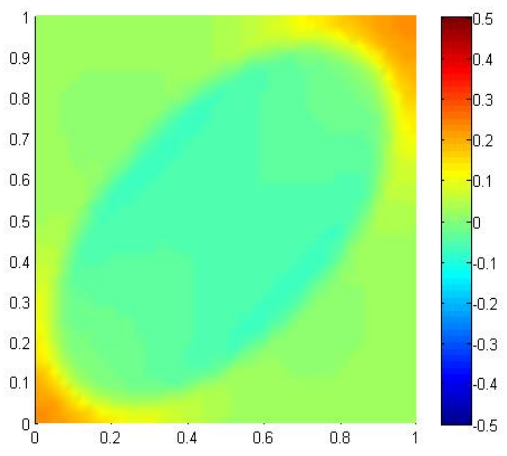

$u_{e, p o d}$

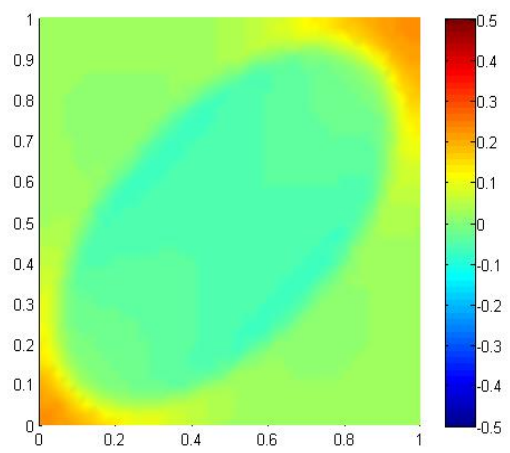

Figure 2: Top (respectively, bottom): Snapshots of the full finite element (respectively POD) solution at time $20 \mathrm{~ms}$ computed using the coupling method. at the depolarization phase. The action potential distribution is in the left column and the extracellular potential distribution is in the right column.

- Solve the extra-cellular potential :

$$
S_{3} u_{e}^{n+1}=-S_{2} V_{m}^{n+1}
$$

The stability of this Gauss-Seidel-like and a Jacobi-like schemes have been proved in [12]. Here we present a numerical scheme that combines both time splitting and POD reduced order methods. Suppose that we have a solution once this problem for a set of parameters, we build the new snapshot matrix by concatenating the the 
action potential and extracellular potential as follows:

$$
A=\left(\begin{array}{cccc}
V_{m}\left(x_{1}, t_{1}\right) \cdots & V_{m}\left(x_{1}, t_{N_{t}}\right), & u_{e}\left(x_{1}, t_{1}\right) \cdots & u_{e}\left(x_{1}, t_{N_{t}}\right) \\
\vdots & \vdots & \vdots & \vdots \\
\vdots & \vdots & \vdots & \vdots \\
V_{m}\left(x_{M}, t_{1}\right) \cdots & V_{m}\left(x_{M}, t_{N_{t}}\right), & u_{e}\left(x_{M}, t_{1}\right) \cdots & u_{e}\left(x_{M}, t_{N_{t}}\right)
\end{array}\right) \in \mathbb{R}^{M \times 2 N_{t}}
$$

After performing a SVD as explained above, we build a POD basis $\Phi=\left(\phi_{k}\right)_{k=1}^{K}$ where $\phi_{k} \in \mathbb{R}^{M}$ for $k=1, \ldots, M$. Each of the equations is now projected on this new basis and solved using the same strategy as in Section 3.2. The numerical results showing the accuracy of combining the pod method to time splitting scheme is shown in the next paragraph.

\subsection{Sensitivity of the POD solution to the ionic parameter}

In order to assess the robustness of these methods and their sensitivity with respect to the ionic model parameters $\tau_{\text {in }}, \tau_{\text {out }}, \tau_{\text {open }}$ and $\tau_{\text {close }}$, we solve the reduced order model for new values of these parameters using the POD basis constructed in Section 3.1 for the coupling method and the POD basis constructed in section 5.1 for the time splitting approach. Each of the parameters is varied between half and three halves its original value as given in Table 2.

\begin{tabular}{llll}
\hline$\tau_{\text {close }}$ & $\tau_{\text {open }}$ & $\tau_{\text {in }}$ & $\tau_{\text {out }}$ \\
75 & 60 & 0.15 & 3 \\
100 & 80 & 0.2 & 4 \\
125 & 100 & 0.25 & 5 \\
150 & 120 & 0.3 & 6 \\
175 & 140 & 0.35 & 7 \\
200 & 160 & 0.4 & 8 \\
225 & 180 & 0.45 & 9 \\
\hline
\end{tabular}

Table 2: Different values of cell membrane parameters used for the sensitivity analysis

After solving the reduced order problem, we compute the error between the full finite element and the reduced order solutions. In Figures 3 ( respectively 4, 5 and 6) we show the evolution of the $L^{2}$ relative errors of the action potential $V_{m}$ (blue continuous line) and the extra-cellular potential $u_{e}$ (red dashed line) with 
respect to parameter $\tau_{\text {close }}$ (respectively $\tau_{\text {open }}, \tau_{\text {out }}$ and $\tau_{\text {in }}$ ). In each figure the result provided by the coupling method is on the left and the result of the the time splitting approach is on the right. We remark that both the coupling method and the time splitting approach provide the same accuracy for all the parameters. This is in line with the stability result obtained in [12] for the finite element method but also with the stability result (21)-(24). We also remark in Figures (3, 4, 5 and 6) that for each parameter the minimum of $L^{2}$ relative error between the POD solution and the finite element solution is reached at the control values provided in Table1. This is expected because the POD basis was built using the control parameters.

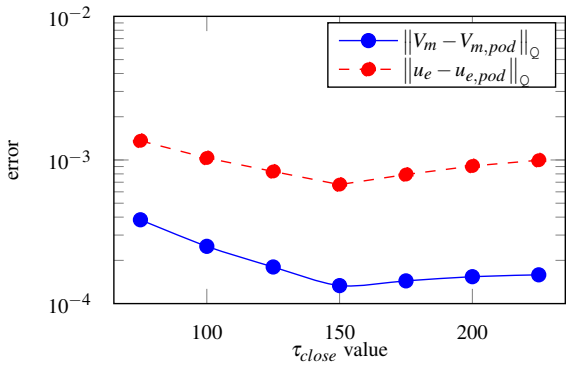

(a)

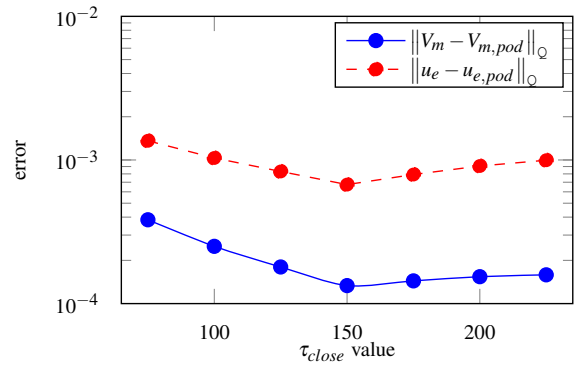

(b)

Figure 3: (a) (respectively, $(b)$ ): The error between the finite elements solution and the POD solution when the value of the paramerter $\tau_{\text {close }}$ vary using full coupling (respectively, GaussSeidel) method. .

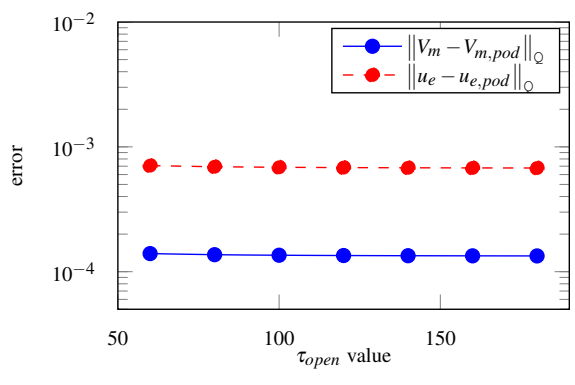

(a)

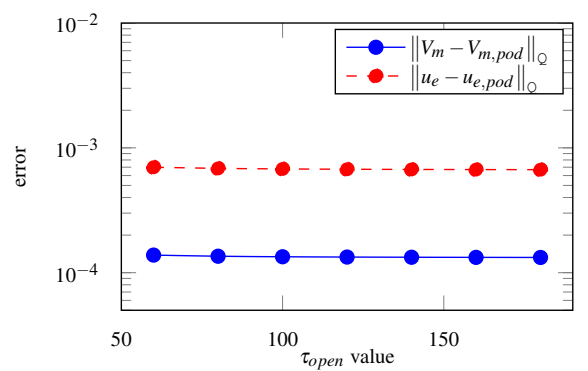

(b)

Figure 4: (a) (respectively, $(b)$ ): The error between the finite elements solution and the POD solution when the value of the paramerter $\tau_{\text {open }}$ vary using full coupling (respectively, GaussSeidel) method.

As concerns the robustness of the POD method with respect to parameters variation, we remark that for parameters $\tau_{c l o s e}, \tau_{\text {open }}$ and $\tau_{\text {out }}$ the relative error is 
less than $1 \%$. However, for parameter $\tau_{\text {in }}$ the error significantly increases when the parameter is far from its original value. In particular for $\tau_{\text {in }}$ less than 0.175 the error is higher than $1 \%$ and it reaches $10 \%$ for $\tau_{i n}=0.15$. This means that POD basis constructed with the original parameters is able to approximate the solution with a good accuracy for different values of parameters $\tau_{\text {close }}, \tau_{\text {open }}$ and $\tau_{\text {out }}$. But for $\tau_{\text {in }}$, the accuracy is acceptable (less than $1 \%$ ) when $\tau_{\text {in }} \in[0.175,0.45]$. In order to cover a bigger interval for the parameter $\tau_{i n}$, the full finite element solution used in the snapshots matrix (i.e, used for the reconstruction of the POD basis) has to be solved for different values of $\tau_{i n}$. These solutions have to be introduced in the snapshots matrix used to construct the POD basis.

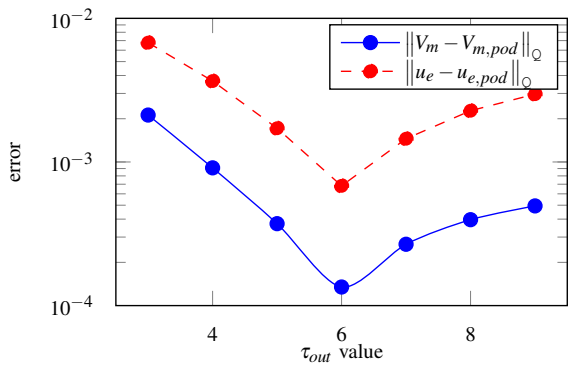

(a)

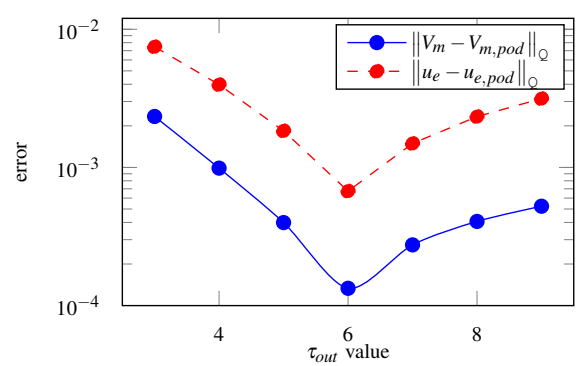

(b)

Figure 5: $(a)$ (respectively, $(b)$ ): The error between the finite elements solution and the POD solution when the value of the paramerter $\tau_{\text {out }}$ vary using full coupling (respectively, Gauss-Seidel) method.

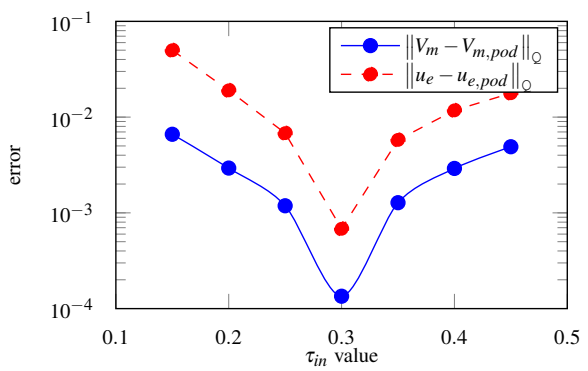

(a)

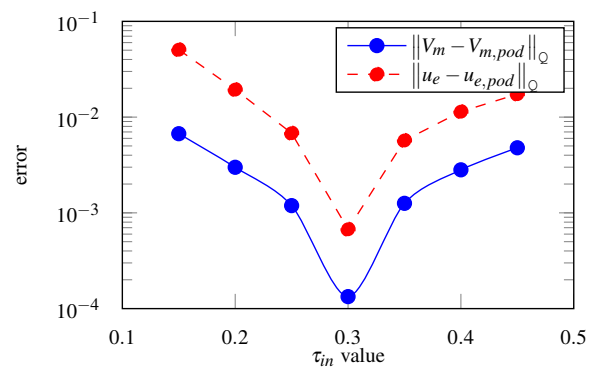

(b)

Figure 6: (a) (respectively, $(b)$ ): The error between the finite elements solution and the POD solution when the value of the paramerter $\tau_{\text {in }}$ vary using full coupling (respectively, Gauss-Seidel) method. 


\section{3D Numerical results}

In this section we apply the POD method to the case studied in the $\mathrm{N}$-version benchmark paper by Niederer et al. [25]. The tissue geometry is defined as a cuboid, with dimensions of $3 \times 7 \times 20 \mathrm{~mm}$ (see Figure 7), fibres are defined parallel the long direction and conductivity tensor is axisymmetric, with values reported in Table 3. In all the simulations performed in this section the space and time steps are respectively $h=0.02 \mathrm{~cm}$ and $d t=0.005 \mathrm{~ms}$, the simulation duration is $600 \mathrm{~ms}$. The stimulus is applied within the cube marked S in Figure 7. The number of nodes in the finite element mesh is 52,500 and the number of degrees of freedom in the linear system to be solved is 105,000 .

\begin{tabular}{cccc}
\hline$\sigma_{\mathrm{i}}^{\mathrm{l}}[\mathrm{mS} / \mathrm{cm}]$ & $\sigma_{\mathrm{i}}^{\mathrm{t}}[\mathrm{mS} / \mathrm{cm}]$ & $\sigma_{\mathrm{e}}^{\mathrm{l}}[\mathrm{mS} / \mathrm{cm}]$ & $\sigma_{\mathrm{e}}^{\mathrm{t}}[\mathrm{mS} / \mathrm{cm}]$ \\
\hline 1.3342 & 0.17606 & 4.0025 & 2.1127 \\
\hline
\end{tabular}

Table 3: Adopted value for the intra and extracellular conductivities in fiber and cross-fiber directions.

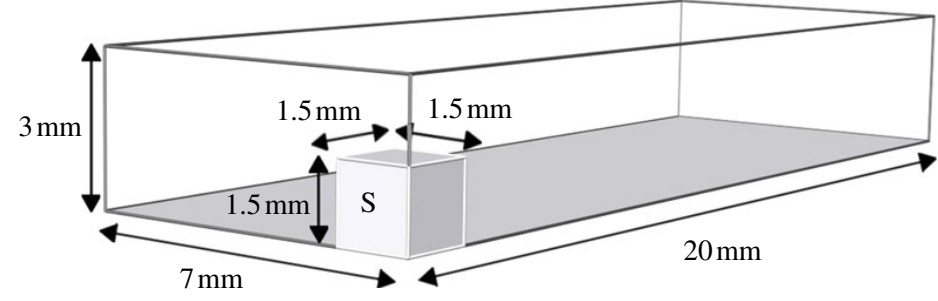

Figure 7: Schematic representation of the computational domain. Reproduced from paper [25].

We then present a study on the usefulness of the POD method when applied to the massively parallel code Chaste and we show the gain in terms of computational cost and the scalability of this approach. We also use the physiologically detailed human cardiomyocyte ionic model developed by Ten Tusscher and Panfilov [31] This model consists of of 18 state variables some of them represent gate variables and others concentrations of different ionic entities and one variable represents the action potential.

\subsection{Choice of the POD basis}

In order to test the reduced order model, we first solve the full finite elements problem. Then, from the time snapshots of the full solution we extract the POD 
basis as described in section 3.1. The number of the POD modes is chosen to be equal to $N$ when the $N$ first eigen modes are able to represent the full finite element solution up to the tolerance that we choose in terms of energy. In this work we truncate the $N$ first modes when the relative error $\mathscr{E}$ of the POD solution is less than the chosen tolerance.

$$
\mathscr{E}=\sqrt{\frac{\int_{0}^{T}\left\|u(x, t)-u_{P O D}^{k}(x, t)\right\|_{L^{2}(\Omega)} d t}{\int_{0}^{T}\|u(x, t)\|_{L^{2}(\Omega)} d t}}
$$

In Figure $8(a)$, we plot the relative error $\mathscr{E}$ as function of $N$ representing the $N$ first eigen modes. Fixing the tolerance to 0.01 , we remark that 200 modes are sufficient in order to obtain a POD solution accurate up to a tolerance of $1 \%$. In fact, adopting 200 modes, the relative error between the reduced order solution and the full problem one is of $0.46 \%$.

In Figure $8(b)$, we plot the computational time (per time step) with respect to the number of modes. We remark that this dependence is linear and we provide the linear fitting of this curve obtained using by least square (red line). The straightline equation of the least square fitting is given by $(y=0.0241 x-0.3884)$. In the same figure we also report the computational time required per time iteration and the corresponding number of mode. This reflects the linearity of the computational time to the number of modes in the POD basis. Since the inverse of the reduced problem matrix is computed once for all. The only additional cost when increasing the number of modes forming the POD basis arises from the additional scalar products in the projection of the RHS (mainly the term of the ionic current) and the reconstruction of the solution phase. Hence, if for specific applications, $1 \%$ of error is not sufficiently accurate, one should take a higher number of modes. 


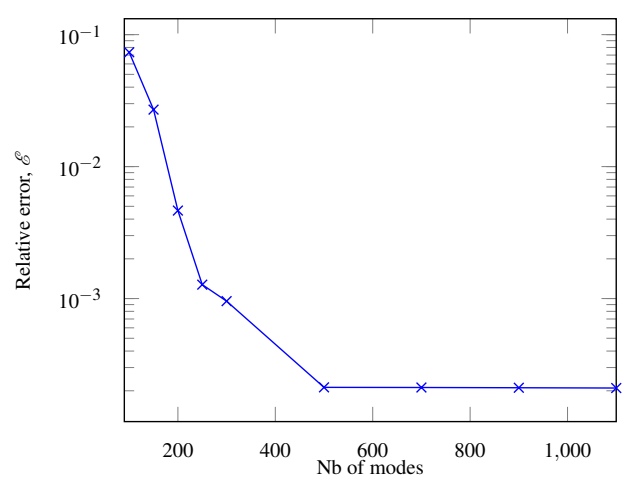

(a)

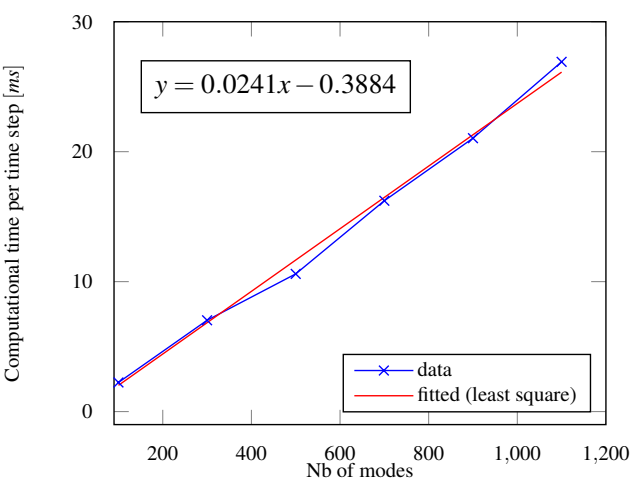

(b)

\begin{tabular}{rcccccc}
\hline Nb of modes & 100 & 300 & 500 & 700 & 900 & 1100 \\
\hline Computational time & 2.24 & 7.03 & 10.6 & 16.23 & 21.04 & 26.91 \\
\hline
\end{tabular}

Figure 8: $(a)$ : Evolution of the energy relative error $\mathscr{E}$ in terms of number of modes for the bidomain problem. $(b)$ : Evolution of Computational cost per time iteration in terms of number of modes for the biodomain problem. (blue line, bottom table) and the least square fitting (red line).

For the sake of illustration, we show in Figure 9 a snapshot of the 3D solution of the action potential for the full bidomain and POD solutions at the depolarization phase $(25 \mathrm{~ms})$. We clearly see that wave fronts are well synchronized.

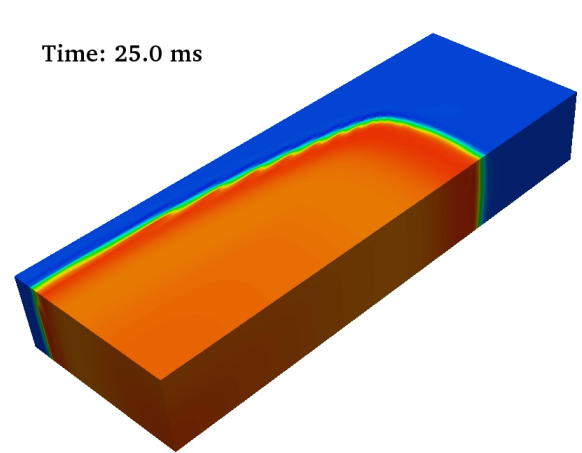

(a)

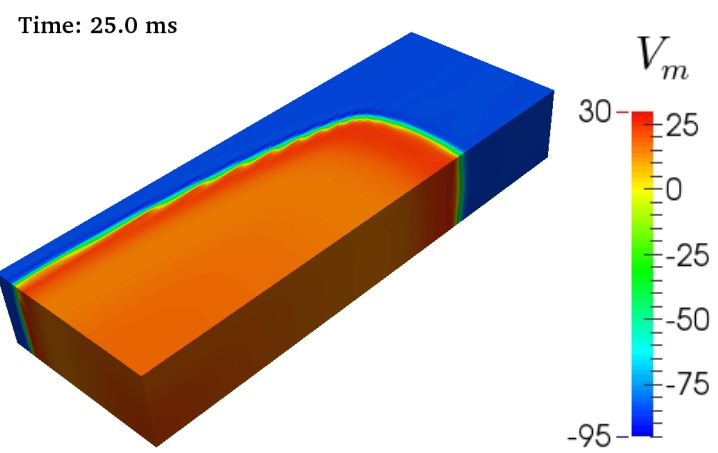

(b)

Figure 9: Snapshot of the action potential computed with the full finite element model $(a)$ and the reduced order bidomain model using 200 modes $(b)$ at time $25 \mathrm{~ms}$. 


\subsection{Scalability of the POD method}

In this paragraph we propose to test the scalability of the reduced order method. We make use of the "Plateforme Fédérative pour la Recherche en Informatique et Mathématiques" (PLAFRIM ${ }^{1}$ ), more details about the machine on which we run the simulations could be found in its web page ${ }^{2}$. We first fix the number of modes to 200. According to the previous paragraph, 200 modes are sufficient to achieve an accuracy of $1 \%$ in term of energy. We solve the bidomain model using both POD and full finite element methods on multiple processors spanning from 1 to 384 CPUs. We first compare the gain in terms of time. In Figure $10(a)$, we compare the computational time required for solving one heart beat between the full and the reduced order simulations while increasing the number of processors. We remark that the full order bidomain solution computational cost decreases with the number of processors until 128. Then for a higher number of processors, we couldn't get any improvement. Even worst, the computational time starts to increase with the number of processors when it is higher than 264. On the contrary, in the reduced order case the computational time still decreases importantly with the number of processors.

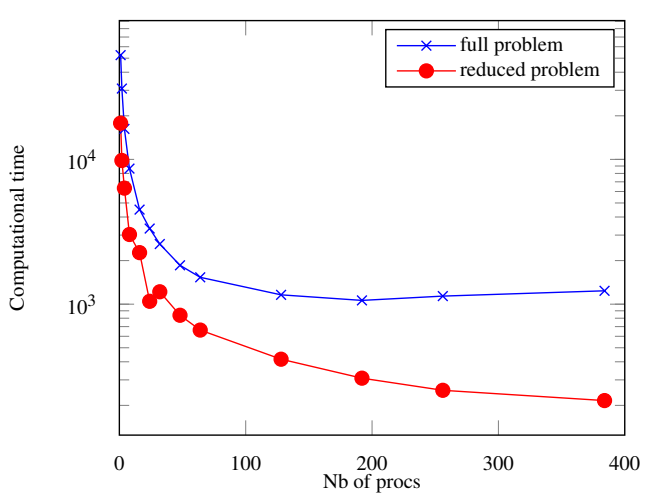

(a)

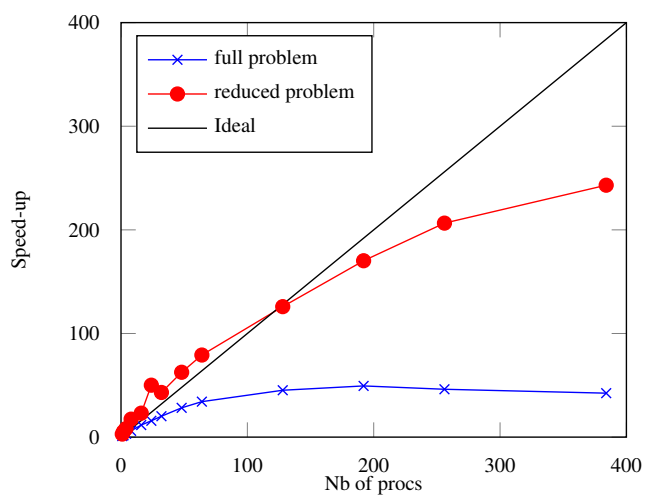

(b)

Figure 10: $(a)$ : Comparison between the full (blue) and the reduced order (red) bidomain solution computation cost. $(b)$ : Comparison between the full (blue) and the reduced order (red) bidomain solution speed up, when the reference computation time is given by the full order solver. The improvement provided by the reduced order method could be seen as the difference between the red and the blue lines.

\footnotetext{
${ }^{1}$ https://plafrim.bordeaux.inria.fr/

${ }^{2}$ https://plafrim.bordeaux.inria.fr/doku.php?id=plateforme:configurations:fourmi
} 
Using one processor the reduced order solver is 3 times faster than the full order solver while it is 8 times faster when using 384 processors for both solvers. In Figure $10(b)$, we show the speed up curves for both methods, defined as the computation time of the full order problem on one CPU over the computation time of the full and reduced order problems on multiple CPUs. In agreement with the results shown for the computational time, we see that the speed up curve of the full order solver increases until 128 processors and starts to decrease from 264 processors; as far as the reduced order solver is concerned, we remark a superlinear speed up between 1 and 128 CPUs, and it increases monotonously until 384 CPUs. If we compare the gain that we obtained by combining HPC and reduced order problem, since the time cost of the full order problem using one processor is 52,500 seconds and the time cost of reduced order solution using 384 processors is 216 seconds, we obtain a speed-up of 243. In this case, solving the bidomain problem with the POD method is six times faster than the full finite element.

\section{Conclusion}

We have presented in this work a reduced order approach based on POD method for the computation of the electrical activity of the heart. Our main finding in this paper is the proof of the stability of the POD method based on an a priori error estimate. This theoretical result shows that we can control the gap between the full finite element solution and the POD solution of the bidomain equation by controlling the gap between the finite element solution and its projection on the used POD basis. We also showed that the POD method could be used for different strategies of solving the bidomain model. It could be used for a fully coupled scheme or by using a time splitting schemes. The numerical results show that it is stable in both cases. In order to evaluate the usefulness of this approach in parameter estimation problem, we conduct numerical simulations in a $2 \mathrm{D}$ case. We build a POD basis using the original parameters of the ionic model and we computed the $L^{2}$ relative error between the finite elements solution and the reduced order solution for different parameters. We conclude that in case of parameter estimation framework it is recommended to use the POD in order to estimate $\tau_{\text {close }}$, $\tau_{\text {open }}$ and $\tau_{\text {out }}$. But to estimate $\tau_{\text {in }}$, the data from which the POD basis is computed should be sufficiently rich in order to maintain a good accuracy of the results. We also studied the scalability of the POD method and compare it to the scalability of the full finite element method using a 3D model. Our results show that using 384 CPUs we obtain a speed up of 243 for the POD method and only 42 using the full finite element method. 


\section{Acknowledgements}

This work has been supported by EPICARD cooperative research program, funded by INRIA international laboratory LIRIMA. The LAMSIN researchers work is supported on a regular basis by the Tunisian Ministry for Higher Education, Scientific Research and Technology. 3D experiments presented in this paper were carried out using the PLAFRIM experimental testbed, being developed under the Inria PlaFRIM development action with support from Bordeaux INP, LABRI and IMB and other entities: Conseil Rgional d'Aquitaine, Universit de Bordeaux and CNRS (and ANR in accordance to the programme d?investissements d?Avenir (see http://www.plafrim.fr/). We also thank the anonymous reviewers for their recommandations that helped us to improve the quality of the paper.

[1] T.M. Austin, M.L. Trew, and A.J. Pullan. Solving the cardiac bidomain equations for discontinuous conductivities. IEEE Trans. Biomed. Eng., 53(7):1265-72, 2006.

[2] M. Boulakia, E. Schenone, and J.F. Gerbeau. Reduced-order modeling for cardiac electrophysiology, application to parameter identification. International Journal for Numerical Methods in Biomedical Engineering, 28(6-7), 2012.

[3] Muriel Boulakia, Serge Cazeau, Miguel A Fernández, Jean-Frédéric Gerbeau, and Nejib Zemzemi. Mathematical modeling of electrocardiograms: a numerical study. Annals of biomedical engineering, 38(3):1071-1097, 2010.

[4] Muriel Boulakia, Miguel A Fernández, Jean-Frédéric Gerbeau, and Nejib Zemzemi. Numerical simulation of electrocardiograms. In Modeling of Physiological Flows, pages 77-106. Springer, 2012.

[5] Muriel Boulakia, Miguel Angel Fernández, Jean-Frédéric Gerbeau, and Nejib Zemzemi. A coupled system of pdes and odes arising in electrocardiograms modeling. Applied Mathematics Research eXpress, 2008:abn002, 2008.

[6] Yves Bourgault, Yves Coudiere, and Charles Pierre. Existence and uniqueness of the solution for the bidomain model used in cardiac electrophysiology. Nonlinear analysis: Real world applications, 10(1):458-482, 2009.

[7] Dominique Chapelle, Asven Gariah, and Jacques Sainte-Marie. Galerkin approximation with proper orthogonal decomposition: new error estimates and illustrative examples. ESAIM: Mathematical Modelling and Numerical Analysis, 46(04):731757, 2012.

[8] Piero Colli Franzone and Luca F Pavarino. A parallel solver for reaction-diffusion systems in computational electrocardiology. Mathematical models and methods in applied sciences, 14(06):883-911, 2004. 
[9] Yves Coudiere, Charles Pierre, Olivier Rousseau, and Rodolphe Turpault. A 2d/3d discrete duality finite volume scheme. application to ecg simulation. International Journal On Finite Volumes, 6(1):1-24, 2009.

[10] Hüsnü Dal, Serdar Göktepe, Michael Kaliske, and Ellen Kuhl. A fully implicit finite element method for bidomain models of cardiac electromechanics. Computer methods in applied mechanics and engineering, 253:323-336, 2013.

[11] J Delville, L Ukeiley, L Cordier, JP Bonnet, and M Glauser. Examination of largescale structures in a turbulent plane mixing layer. part 1. proper orthogonal decomposition. Journal of Fluid Mechanics, 391:91-122, 1999.

[12] Miguel A Fernández and Nejib Zemzemi. Decoupled time-marching schemes in computational cardiac electrophysiology and ecg numerical simulation. Mathematical biosciences, 226(1):58-75, 2010.

[13] Thibault Henri and Jean-Pierre Yvon. Convergence estimates of pod-galerkin methods for parabolic problems. In John Cagnol and Jean-Paul Zolsio, editors, System Modeling and Optimization, volume 166 of IFIP International Federation for Information Processing, pages 295-306. Springer US, 2005.

[14] Craig S Henriquez. Simulating the electrical behavior of cardiac tissue using the bidomain model. Critical reviews in biomedical engineering, 21(1):1-77, 1992.

[15] Sunil M Kandel. The electrical bidomain model: A review. Scholars Academic Journal of Biosciences, 3(7):633-639, 2015.

[16] Pierre Kerfriden, Pierre Gosselet, Sondipon Adhikari, and Stephane Pierre-Alain Bordas. Bridging proper orthogonal decomposition methods and augmented newton-krylov algorithms: an adaptive model order reduction for highly nonlinear mechanical problems. Computer Methods in Applied Mechanics and Engineering, 200(5):850-866, 2011.

[17] K Kunisch and Stefan Volkwein. Galerkin proper orthogonal decomposition methods for a general equation in fluid dynamics. SIAM Journal on Numerical analysis, 40(2):492-515, 2002.

[18] Karl Kunisch and Aurora Marica. Well-posedness for the mitchell-scheaffer model of the cardiac membrane. Technical Report SFB-Report No. 2013-018, KarlFranzens Universitat Graz, A-8010 Graz, Heinrichstrasse 36, Austria, December 2013. 
[19] Karl Kunisch and Marcus Wagner. Optimal control of the bidomain system (iv): Corrected proofs of the stability and regularity theorems. arXiv preprint arXiv:1409.6904, 2014.

[20] G.T. Lines, P. Grøttum, and A. Tveito. Modeling the electrical activity of the heart: a bidomain model of the ventricles embedded in a torso. Comput. Vis. Sci., 5(4):195213, 2003.

[21] Gary R Mirams, Christopher J Arthurs, Miguel O Bernabeu, Rafel Bordas, Jonathan Cooper, Alberto Corrias, Yohan Davit, Sara-Jane Dunn, Alexander G Fletcher, Daniel G Harvey, et al. Chaste: an open source c++ library for computational physiology and biology. PLoS computational biology, 9(3):e1002970, 2013.

[22] Colleen C Mitchell and David G Schaeffer. A two-current model for the dynamics of cardiac membrane. Bulletin of mathematical biology, 65(5):767-793, 2003.

[23] R. Guyonnet N. Aubry and R. Lima. Spatio-temporal analysis of complex signals : theory and applications. J. Statis. Phys, 64(3/4):683-739, 1991.

[24] Chamakuri Nagaiah, Karl Kunisch, and Gernot Plank. On boundary stimulation and optimal boundary control of the bidomain equations. Math Biosci., 245(2):206-215, 2013.

[25] S.A. Niederer, E. Kerfoot, A.P. Benson, M.O. Bernabeu, O. Bernus, C. Bradley, E.M. Cherry, R. Clayton, F.H. Fenton, A. Garny, et al. Verification of cardiac tissue electrophysiology simulators using an n-version benchmark. Philosophical Transactions of the Royal Society A: Mathematical, Physical and Engineering Sciences, 369(1954):4331-4351, 2011.

[26] J.L. Lumley P. Holmes and G. Berkooz. Turbulence, Coherent Structures, Dynamical Systems and Symmetry. Cambridge Monographs on Mechanics, 1996.

[27] Mark Potse, Bruno Dubé, Jacques Richer, Alain Vinet, and Ramesh M Gulrajani. A comparison of monodomain and bidomain reaction-diffusion models for action potential propagation in the human heart. Biomedical Engineering, IEEE Transactions on, 53(12):2425-2435, 2006.

[28] Muruhan Rathinam and Linda R Petzold. A new look at proper orthogonal decomposition. SIAM Journal on Numerical Analysis, 41(5):1893-1925, 2003.

[29] J. Sundnes, G.T. Lines, and A. Tveito. Efficient solution of ordinary differential equations modeling electrical activity in cardiac cells. Math. Biosci., 172(2):55-72, 2001. 
[30] Joakim Sundnes, Glenn Terje Lines, Xing Cai, Bjørn Frederik Nielsen, Kent-Andre Mardal, and Aslak Tveito. Computing the electrical activity in the heart, volume 1. Springer Science \& Business Media, 2007.

[31] KHWJ Ten Tusscher and AV Panfilov. Cell model for efficient simulation of wave propagation in human ventricular tissue under normal and pathological conditions. Physics in medicine and biology, 51(23):6141, 2006.

[32] Mark L Trew, Bruce H Smaill, David P Bullivant, Peter J Hunter, and Andrew J Pullan. A generalized finite difference method for modeling cardiac electrical activation on arbitrary, irregular computational meshes. Mathematical biosciences, 198(2):169-189, 2005.

[33] Leslie Tung. A bi-domain model for describing ischemic myocardial dc potentials. $\mathrm{PhD}$ thesis, Massachusetts Institute of Technology, 1978.

[34] Edward J Vigmond, Felipe Aguel, Natalia Trayanova, et al. Computational techniques for solving the bidomain equations in three dimensions. Biomedical Engineering, IEEE Transactions on, 49(11):1260-1269, 2002.

[35] E.J. Vigmond, R. Weber dos Santos, A.J. Prassl, M Deo, and G. Plank. Solvers for the cardiac bidomain equations. Progr. Biophys. Molec. Biol., 96(1-3):3-18, 2008.

[36] Stefan Volkwein. Model reduction using proper orthogonal decomposition. Lecture Notes, Institute of Mathematics and Scientific Computing, University of Graz. see http://www. uni-graz. at/imawww/volkwein/POD. pdf, 2011.

[37] Zhu Wang, Imran Akhtar, Jeff Borggaard, and Traian Iliescu. Proper orthogonal decomposition closure models for turbulent flows: a numerical comparison. Computer Methods in Applied Mechanics and Engineering, 237:10-26, 2012.

[38] Néjib Zemzemi. Étude théorique et numérique de lactivité électrique du cœur: Applications aux électrocardiogrammes. $\mathrm{PhD}$ thesis, Paris 11, 2009. 